\title{
REFLEXIVE COMPACT MAPPINGS ${ }^{1}$
}

\author{
EDWIN DUDA
}

1. Introduction. A mapping $f$ from one topological space $X$ to another $Y$ is called reflexive compact provided that $f^{-1} f(A)$ is compact for every compact subset $A$ of $X$. We prove that this condition characterizes mappings that generate an upper-semicontinuous (u.s.c.) decomposition. We show that a class of mappings that generalize $\epsilon$-mappings is a class of reflexive compact mappings. We also show how reflexive compactness of a mapping implies compactness of a mapping. Finally as an application we prove a result about mappings on the plane which generalizes theorems of [8], [9], [12].

2. Notation and definitions. Throughout $X$ and $Y$ will represent topological Hausdorff spaces and $f$ will be mapping (continuous function) of $X$ into $Y$. A mapping $f$ of $X$ into $Y$ is said to generate an u.s.c. decomposition of $X$ if for every open set $U$ of $X$, the union $V$ of point inverses $f^{-1}(y)$ contained in $U$ is an open subset of $X$; see [1], [7]. A mapping is monotone if point inverses are compact and connected. A mapping is compact if the inverse image of each compact set is compact. A topological space $X$ is a $k$-space if a subset $C$ of $X$ intersects each compact set $D$ in a closed set implies $C$ is a closed set, [6]. A mapping on a metric space is an $\epsilon$-mapping if all point inverses have diameter less than $\epsilon$.

3. Upper-semicontinuity. In this section we obtain a characterization of mappings with compact point inverses that generate an u.s.c. decomposition. As in [11] every closed mapping generates an u.s.c. decomposition. Also, if $f$ is a mapping from a locally compact space $X$ to a space $Y$ with components of point inverses compact, then the decomposition of $X$ into components of point inverses is u.s.c.; see [2], [9], [11]. In particular, a monotone mapping on a locally compact space generates an u.s.c. decomposition.

THEOREM 1. Let $X$ be a k-space and $f$ a mapping of $X$ into $Y$. If $f$ is a reflexive compact mapping then $f$ generates an upper-semicontinuous decomposition.

Proof. Let $U$ be an open set in $X$ containing a point inverse and let $V$ be the union of the point inverses contained in $U$. We show

Received by the editors July 26, 1965.

1 Supported by National Science Foundation Grant GP 4769. 
$\bar{U}-V$ is closed in $X$ which in turn implies $V$ is open in $X$ and consequently $f$ generates an u.s.c. decomposition. Let $C$ be a compact set in $X$ such that $H=(\bar{U}-V) \cap C \neq \varnothing$. For each point $x$ in $H, f^{-1} f(x)$ $\cap(X-U) \neq \varnothing$. Thus we obtain

$$
f^{-1} f(H) \cap(X-U)=f^{-1} f(C \cap \bar{U}) \cap(X-U) .
$$

The right member is compact which implies compactness of the left member. Denoting the set in line (1) above by $M$ we obtain

$$
f^{-1} f(M) \cap \bar{U}=f^{-1} f(C \cap \bar{U}) \cap(\bar{U}-V) .
$$

The left member is compact and hence so is the right member. The set $C \cap(\bar{U}-V)$ is closed for it is the intersection of the compact sets $C$ and $f^{-1} f(C \cap \bar{U}) \cap(\bar{U}-V)$. Thus $X$ being a $k$-space implies $\bar{U}-V$ is a closed subset of $X$.

It is established in [6] that every locally compact or first countable Hausdorff space is a $k$-space.

Corollary 1. Let $X$ be a locally compact or first countable space and $f$ a mapping of $X$ into $Y$. If $f$ is a reflexive compact mapping, then $f$ generates an u.s.c. decomposition.

We now prove a theorem which implies the converse of Theorem 1 is true. Note that the property of being a $k$-space is not needed in the following theorem.

Theorem 2. Let $f$ be a mapping with compact point inverses of a space $X$ into a space $Y$. If $f$ generates an upper-semicontinuous decomposition, then $f$ is reflexive compact.

Proof. Let $\left\{U_{\alpha}\right\}, \alpha \in \Gamma$, be an open cover of $f^{-1} f(A)$, where $A$ is compact subset of $X$. For each $x$ in $A$, there is some finite number of the $U_{\alpha}$ that cover $f^{-1} f(x)$. Denote the union of one of these finite collections covering $f^{-1} f(x)$ by $V_{x}$. By u.s.c. of the decomposition of $X$ into point inverses the union $W_{x}$ of the point inverses contained in $V_{x}$ is an open set with the property that if $y \in W_{x} \cap A$, then $f^{-1} f(y) \subset W_{x}$. The collection $\left\{W_{x}\right\}, x \in A$, is an open cover of $A$ so there exists a finite number $W_{x_{1}}, W_{x_{2}}, \cdots, W_{x_{n}}$, that cover $A$. The $\left\{W_{x_{i}}\right\}$, $i=1,2, \cdots, n$, also cover $f^{-1} f(A)$ and with each $W_{x_{i}}$ there is associated a finite number of $U_{\alpha}$. These $n$ finite collections of $U_{\alpha}$ give the required finite open cover of $f^{-1} f(A)$.

THEOREM 3. Let $f$ be a mapping with compact point inverses of a $k$ space $X$ into a space $Y$. The mapping $f$ generates an upper-semicontinuous decomposition if and only if $f$ is reflexive compact. 
A characterization of all mappings $f$ that generate an uppersemicontinuous decomposition that is independent of the spaces involved is to simply require that $f^{-1} f(C)$ be a closed subset of the domain space whenever $C$ is a closed subset of the domain space. However, for the applications of this paper the result of Theorem 3 is needed.

The characterization in Theorem 3 can be generalized to mappings with noncompact point inverses reasonably well if the domain space is required to be locally compact. (See [4].) In order to discuss this generalization the following definition is needed. Let $\left\{F_{\alpha}\right\}$ be a collection of closed subsets of a locally compact space $X$. The collection $\left\{F_{\alpha}\right\}$ is said to be scattered if every compact set $A$ in $X$ meets only finitely many of the $F_{\alpha}$. Now if $G$ is an upper-semicontinuous decomposition of $X$ into closed sets, then by the results of [4], the collection of noncompact elements of $G$ must be scattered and their union $F$ is a closed set. Thus, if $f$ is a mapping of a locally compact space $X$ into a space $Y$ that generates an u.s.c. decomposition and $F$ is the union of the noncompact point inverses then $f$ restricted to $X-F$ is reflexive compact. Conversely if $f$ is a mapping such that the set of noncompact point inverses is scattered and $f$ restricted to the complement of the union of the noncompact point inverses is reflexive compact, then $f$ generates an u.s.c. decomposition.

4. $\epsilon$-mappings. In a metric space $X$ let $\rho$ be the distance function and for any set $A$ in $X$, let $\delta(A)$ be the diameter of $A$. The condition on $f$ in the following theorem is a generalization of a condition imposed in [8].

THEOREM 4. Let $f$ be a mapping of a metric space $X$ into a space $Y$. If for every sequence of distinct points $x_{n}$ with no limit point and $y_{0}$ in $X$

$$
\liminf \frac{\delta\left(f^{-1} f\left(x_{n}\right)\right)}{\rho\left(y_{0}, x_{n}\right)}<1,
$$

then $f$ is reflexive compact.

Proof. Clearly the condition implies point inverses must be compact. Suppose $f^{-1} f(A)$ is not compact for some compact set $A$. There exists a sequence of distinct points $\left\{x_{n}\right\}$ in $f^{-1} f(A)$ with no limit point. For each positive integer $n$, let $y_{n}$ be a point of $f^{-1} f\left(x_{n}\right) \cap A$. We can suppose that $\left\{y_{n}\right\}$ converges to a point $y_{0}$ in $A$. For $n$ sufficiently large we have 


$$
\frac{\delta\left(f^{-1} f\left(x_{n}\right)\right)}{\rho\left(y_{0}, x_{n}\right)} \geqq \frac{\delta\left(f^{-1} f\left(x_{n}\right)\right)}{\rho\left(y_{0}, y_{n}\right)+\rho\left(y_{n}, x_{n}\right)}=\frac{\frac{\delta\left(f^{-1} f\left(x_{n}\right)\right)}{\rho\left(y_{n}, x_{n}\right)}}{\frac{\rho\left(y_{0}, y_{n}\right)}{\rho\left(y_{n}, x_{n}\right)}+1} \geqq \frac{1}{\frac{\rho\left(y_{0}, y_{n}\right)}{\rho\left(y_{n}, x_{n}\right)}+1} .
$$

Since $\lim _{n \rightarrow \infty} \rho\left(y_{0}, y_{n}\right)=0$ and $\rho\left(y_{n}, x_{n}\right)$ is bounded away from zero for $n$ sufficiently large, it follows that

$$
\liminf _{n \rightarrow \infty} \frac{\delta\left(f^{-1} f\left(x_{n}\right)\right)}{\rho\left(y_{0}, x_{n}\right)} \geqq 1 .
$$

CoRollary 1. If $f$ is an $\epsilon$-mapping on a metric space $X$ in which closed and bounded sets are compact into a space $Y$, then $f$ is reflexive compact.

5. Compact mappings. Let $f$ be a mapping of $X$ into $Y$. A set $B$ of $X$ is an inverse set of $f$ if $f^{-1} f(B)=B$. A mapping is quasi-compact provided that the image of every open inverse set is an open set [11]. The following theorem and its corollaries relate reflexive compactness of a mapping to compactness [11], [12].

Theorem 5. Let $f(X)=Y$ be a mapping, where $X$ is a k-space. If $f$ is quasi-compact and reflexive compact, then $f$ is a compact mapping.

Proof. Let $\left\{U_{\alpha}\right\}, \alpha \in \Gamma$, be an open cover of $f^{-1}(K)$, where $K$ is a compact subset of $Y$. By Theorem $1, f$ generates an u.s.c. decomposition of $X$. As in the proof of Theorem 2, the cover $\left\{U_{\alpha}\right\}$ can be related to an open cover $\left\{V_{y}\right\}, y \in K$, of $f^{-1} f(K)$, where each $V_{y}$ is an open inverse set contained in the union of a finite number of $U_{\alpha}$. Thus $\left\{f\left(V_{y}\right)\right\}, y \in K$, is an open cover of $K$ and by compactness of $K$ there is some finite number $f\left(V_{y_{1}}\right), f\left(V_{y_{2}}\right), \cdots, f\left(V_{y_{n}}\right)$, that cover $K$. The $V_{y_{1}}, V_{y_{2}}, \cdots, V_{y_{n}}$ cover $f^{-1}(K)$ and hence some finite number of the $U_{\alpha}$ also cover $K$.

Every open or closed mapping is a quasi-compact mapping, hence with the same conditions on the spaces that appear in Theorem 5 , we obtain the following corollary.

COROLlARY 1. If $f$ is an open (closed) mapping and reflexive compact, then $f$ is a compact mapping.

We have already observed that every monotone mapping on a locally compact space generates an u.s.c. decomposition. Thus, each such mapping is reflexive compact. Furthermore, it is not difficult to show that local compactness is a necessary condition on the domain 
space. We obtain immediately the following corollaries.

Corollary 2. If $f(X)=Y$ is a monotone quasi-compact mapping, where $X$ is locally compact, then $f$ is a compact mapping.

Corollary 3. If $f(X)=Y$ is a monotone open (closed) mapping, where $X$ is locally compact, then $f$ is a compact mapping.

6. Plane mappings. In [3] K. Borsuk shows that every $\epsilon$-mapping $f$ of Euclidean $n$-space $E^{n}$ into $E^{n}$ is a compact mapping of $E^{n}$ onto $f\left(E^{n}\right)$. In [8] K. A. Sitnikov generalizes Borsuk's result by replacing $\epsilon$-mappings with a condition that is more restrictive than that of Theorem 4. In [12] G. T. Whyburn shows that every monotone mapping of the plane onto the plane is a compact mapping. The last two results are independent for mappings of the plane on to the plane. In this section we propose to show that every reflexive compact mapping of the plane onto the plane is a compact mapping.

A locally connected generalized continuum is a locally connected, locally compact, connected separable metric space. Let $f(X)=Y$ be a reflexive compact mapping, where $X$ and $Y$ are locally connected generalized continua. Then, as we have seen, $f$ generates an u.s.c. decomposition of $X$. Let $M$ be the decomposition space determined and $\pi$ the natural mapping from $X$ on to $M$. It is well known [10], [11], that $M$ is a locally connected generalized continuum and that $\pi$ is a compact mapping. Also true is that there is a 1-1 mapping $h$ of $M$ onto $Y$ such that $f(x)=h \pi(x)$ for all $x$ in $X$.

Theorem 6. Let $f(X)=E^{2}$ be a mapping, where $X$ is a locally connected generalized continuum with the property that the complement of every compact subset $K$ of $X$ has only one nonconditionally compact component. If $f$ is reflexive compact then $f$ is a compact mapping.

Proof. Let $M$ be the decomposition space generated by $f, \pi$ the natural mapping and $h$ the mapping of $M$ onto $E^{2}$ with the property that $f(x)=h \pi(x)$ for all $x$ in $X$. Since $\pi$ is a compact mapping it follows that the complement of every compact set $K$ of $M$ has only one nonconditionally compact component. Thus, by the Theorem of [5] $h$ must be a homeomorphism. Since $f$ is the composition of two compact mappings it is also a compact mapping.

THEOREM 7. If $f\left(E^{2}\right)=E^{2}$ is a reflexive compact mapping, then $f$ is a compact mapping.

There is an example of a monotone map of $E^{2}$ in to the unit circle $S^{1}$ which is not a compact mapping. Furthermore an example of $\mathrm{K}$. 
Whyburn in [13] shows that the method used in this paper to prove Theorem 7 may not be extendible to higher dimensional Euclidean spaces.

\section{REFERENCES}

1. P. Alexandroff and H. Hopf, Topologie, Springer, Berlin, 1935.

2. H. Bauer, Verallgemeinerung eines Faktorisierungssatzes von G. T. Whyburn, Arch. Math. 10 (1959), 373-378.

3. K. Borsuk, Über Stetige Abbildungen der Euklidischen Raume, Fund. Math. 21 (1933), 236-243.

4. E. Duda, A locally compact metric space is almost invariant under a closed mapping, Proc. Amer. Math. Soc. 16 (1965), 473-475.

5. E. Duda, $A$ theorem on 1-1 mappings, Pacific J. Math. (to appear).

6. J. L. Kelley, General topology, Van Nostrand, New York, 1955.

7. R. L. Moore, Foundations of point set theory, Amer. Math. Soc. Colloq. Publ., Vol. 13, Amer. Math. Soc., Providence, R. I., 1932.

8. K. H. Sitnikov, On continuous mappings of open sets of Euclidean spaces, Mat. Sb. (73) 31 (1952), 439-458.

9. K. Stein, Analytical Zerlegungen-Komplexen Raume, Math. Ann. 132 (1956), 63-93.

10. A. H. Stone, Metrizability of decomposition spaces, Proc. Amer. Math. Soc. 7 (1956), 690-700.

11. G. T. Whyburn, Open and closed mappings, Duke Math. J. 17 (1950), 69-74.

12. - Compactness of certain mappings, Amer. J. Math. 81 (1959), 306-314.

13. K. Whyburn, A nontopological 1-1 mapping onto $E^{3}$, Bull. Amer. Math. Soc. 71 (1965), 533-537.

University of Miami 